\title{
Safety of tirofiban for patients with acute ischemic stroke in routine clinical practice
}

\author{
YUAN-QUN ZHU ${ }^{1}$, YAN-JUN ZHANG ${ }^{2}$, HAI-LIN RUAN ${ }^{3}$, QING LIU $^{2}$, QIN ZHAN $^{2}$ and QIONG LI ${ }^{2}$ \\ ${ }^{1}$ Department of Neurology, Liuzhou Worker's Hospital, The Fourth Affiliated Hospital of Guangxi Medical University, \\ Liuzhou, Guangxi 545005; ${ }^{2}$ Department of Geriatrics, People's Hospital of Zhengzhou, Zhengzhou, Henan 450003; \\ ${ }^{3}$ Department of Emergency, Liuzhou Worker's Hospital, The Fourth Affiliated Hospital of Guangxi Medical University, \\ Liuzhou, Guangxi 545005, P.R. China
}

Received April 11,2014; Accepted December 8, 2014

DOI: 10.3892/etm.2015.2495

\begin{abstract}
The aim of the present study was to investigate the safety of tirofiban alone and in combination with various treatments in acute ischemic stroke (AIS). A total of 120 patients with AIS were included in the study, and these patients were divided into three treatment groups: Group A (tirofiban alone, $\mathrm{n}=68$ ), group B (tirofiban plus thrombolytic therapy, $n=26$ ), and group $\mathrm{C}$ (tirofiban as a 'bridging therapy', $\mathrm{n}=26$ ). Risk factors, stroke severity, initial imaging, treatment regimens, complications and long-term outcomes were analyzed. In total, eight patients $(6.7 \%)$ [six patients $(23.1 \%)$ in group B and two patients $(7.7 \%)$ in group C] had hemorrhage during or subsequent to treatment. Sixteen patients (six in group A, eight in group B and two in group C) succumbed during the hospital admission period. The mortality rate was $13.3 \%$ (8.8\% for group A, 30.7\% for group B and 7.7\% for group C) in the acute phase. A favorable outcome (modified Rankin Scale score, 0-2) in the first three months after stroke was only observed in $43.3 \%$ of patients (44.1\% in group A, $46.7 \%$ in group B and $36.4 \%$ in group C). The mean Barthel index was 72.3 in group A, 84.4 in group B and 56.8 in group C (total patient population, 71.0). The results of the present study have shown that stroke treatment with tirofiban is safe in AIS. A large randomized controlled trial in the future is required to decrease the incidence of the minor bleeding complications associated with tirofiban therapy.
\end{abstract}

Correspondence to: Professor Yan-Jun Zhang, Department of Geriatrics, People's Hospital of Zhengzhou, 33 Yellow River Road, Zhengzhou, Henan 450003, P.R. China

E-mail: yanjunzcn@126.com

Professor Hai-Lin Ruan, Department of Emergency, Liuzhou Worker's Hospital, The Fourth Affiliated Hospital of Guangxi Medical University, 1 Liushi Road, Liuzhou, Guangxi 545005, P.R. China

E-mail: ruanhailinlz@126.com

Key words: tirofiban, acute ischemic stroke, safety

\section{Introduction}

Acute ischemic stroke (AIS) is a common cause of morbidity and mortality worldwide. Thrombolysis with recombinant tissue plasminogen activator (rtPA) is the only proven beneficial therapy in AIS, and this is received by $<2 \%$ of patients (1). The inaccessibility of this treatment to the majority of patients is due to a number of factors: A lack of adequate transport facilities and infrastructure, including facilities for thrombolysis in most centers; the high cost of tPA; and a lack of awareness among the public and doctors (2). Furthermore, there has been a slight increase in the incidence of hemorrhagic complications, such as intracranial hemorrhage and gastrointestinal bleeding associated with thrombolysis $(2,3)$.

Glycoprotein IIb/IIIa inhibitors, following their initial success in patients with acute coronary syndromes, have led to an increasing interest to treat AIS over the past decade (4-8). Highly selective platelet antagonists, the glycoprotein (gp) IIb/IIIa inhibitors, block the fibrin binding receptors reversibly and effectively prevent platelet aggregation.

Bridging therapy is the combination of intravenous (IV) and intra-arterial (IA) thrombolysis, and is part of the therapeutic armamentarium in the daily practice of several stroke centers (9). Some clinicians recommend the use of tirofiban as a bridging therapy during the perioperative period (10). Tirofiban is a fast-acting, highly selective nonpeptide gpIIb/IIIa antagonist used for the treatment of acute coronary syndrome up to $48 \mathrm{~h}$ after onset (6); however, the risk factors associated with tirofiban administration, the impacts of stroke severity and the type of treatment regimen on the efficacy of the drug and the exact effect of tirofiban on patients with AIS, including complications, incidence of symptomatic or asymptomatic hemorrhage and long-term outcomes, are currently unclear. The aim of the present study was to investigate the safety of tirofiban alone and in combination with various treatments in AIS.

\section{Patients and methods}

Patients. A total of 120 patients with AIS were included in this study for treatment with tirofiban in the context of an individual treatment trial. The study was approved by the Ethics Committee of the People's Hospital of Zhengzhou (Zhengzhou, 
China) in accordance with the Declaration of Helsinki. Written informed consent was obtained from all participants.

Treatment. Patients received an initial dose of $0.4 \mathrm{mg}$ over a 30 min period, and tirofiban administration was then continued at a dosage of $0.1 \mathrm{mg} / \mathrm{kg}$ body weight/h. The recommended infusion duration was $24 \mathrm{~h}$. Patients receiving tirofiban alone and those receiving tirofiban and other recanalization methods were included. The patients were divided into three groups: Group A (n=68; tirofiban monotherapy; Hangzhou Zhongmei Huadong Pharmaceutical Co., Ltd., Hangzhou, China), group B ( $n=26$; tirofiban in combination with IV or intra-arterial IA thrombolysis) and group $\mathrm{C}[\mathrm{n}=26$; tirofiban as a 'bridging therapy', i.e. an additional recanalization measure started prior to and continued during and subsequent to the intervention, known as percutaneous transluminal coronary angioplasty (PTCA) and coronary stent implantation].

Data collection. Vascular risk profile, stroke localization, treatment duration, cumulative dose and the time of tirofiban doses were recorded. The modified Rankin Scale (mRS) (11) was used to describe the severity of the stroke.

Pretreatment imaging, including cerebral computed tomography (CCT) and stroke magnetic resonance imaging (MRI), was performed to evaluate infarct demarcation and any early signs of infarction. In patients who had completed an angiography prior to treatment, it was noted whether a vascular occlusion had occurred.

CCT and MRI images were scored for bleeding complications during and subsequent to tirofiban application. To determine the severity of bleeding, the European Cooperative Acute Stroke Study II criteria (12), as follows, were used: Hemorrhagic infarction 1 (HI1), small hemorrhages along the infarct margins; HI2, confluent hemorrhage within the infarct area but without a space-occupying effect; parenchymal hematoma (PH) 1, bleeding affects $\leq 30 \%$ of the infarct area and may show a small space-occupying effect; $\mathrm{PH} 2$, blood flow affecting $>30 \%$ of the infarct area and with a significant space-occupying effect; symptomatic intracerebral hemorrhage, bleeding in any region of the brain accompanied by a significant clinical deterioration (12). Those patients in whom an initial vascular occlusion was documented were evaluated for recanalization by means of representation-guided vascular CT angiography (CTA), magnetic resonance angiography (MRA) or digital subtraction angiography (DSA) following treatment. The National Institutes of Health Stroke Scale (NIHSS), mRS and Barthel index (BI) were used to assess the outcomes at discharge.

Statistical analysis. All experiments were performed at least three times. Data are expressed as the mean \pm standard deviation. One-way analysis of variance was used to analyze the data. Statistical analyses were carried out using SPSS 17.0 software (SPSS Inc., Chicago, IL, USA). P $<0.05$ was considered to indicate a statistically significant difference.

\section{Results}

General characteristics. The study included a total of 120 patients (70 males and 50 females), who were treated with
Table I. Vascular risk profile of patients.

\begin{tabular}{lc}
\hline Risk factors & $\mathrm{n}(\%)$ \\
\hline Diabetes mellitus & $16(13.3)$ \\
Arterial hypertension & $74(61.7)$ \\
Arteriosclerosis & $68(56.7)$ \\
Heart rhythm disorders & $30(25.0)$ \\
Other cardiac source of embolism & $26(21.7)$ \\
Hyperlipidemia & $52(43.3)$ \\
Obesity & $52(43.3)$ \\
Previous stroke & $28(23.3)$ \\
Smoking (current and former) & $30(25.0)$
\end{tabular}

tirofiban in the context of an individual treatment trial. The vascular risk profile of the patients is summarized in Table I.

Patient groups. The majority of the patients $(n=68)$ received tirofiban as a monotherapy and were assigned to group A. In these patients, clot lysis by tPA was no longer a viable option, primarily due to a lapsed three-hour time window. A total of 18 patients were treated within the time window, meaning that different exclusion criteria for the lysis treatment were applicable. In addition, two patients suffered from ulcerative colitis and alcohol abuse, respectively, and another four patients had already received the tirofiban treatment. These 26 patients were thus assigned to group B and further divided into subgroups according to whether tirofiban was administered following systemic or IA thrombolysis.

The remaining 26 patients were assigned to group C. In these patients, other recanalization or bridging IV therapy was initiated during the tirofiban therapy. In one case, the bridge was combined with a carotid endarterectomy. The young patient had previously been treated systemically due to a medium infarction. Since the symptoms did not improve, the patient was brought to the People's Hospital of Zhengzhou, where a stenosis of the internal carotid artery and a thrombus just distal to the stenosis were found the day after admission. Eight patients received IA thrombolysis, which was performed from the onset of symptoms until $2.5 \mathrm{~h}$ after the start of treatment. In three patients, however, the time of symptom onset was unclear; IA embolectomy was performed in these three patients. The mean time to the initiation of treatment with tirofiban was $6.25 \mathrm{~h}$, with another $35 \mathrm{~min}$ until the beginning of the intervention. In one patient receiving IV lysis and tirofiban simultaneously, the time of treatment was $2.5 \mathrm{~h}$. In all patients undergoing neuro-interventional radiology measures, the infusion was maintained during and following treatment by coronary angiography or by PTCA in the presence or absence of stent implantation in patients with acute coronary syndrome. The distribution of stroke localization in the different groups is shown in Table II.

Imaging findings. Prior to treatment, all of the patients received CCT; in two cases, MRI was performed to rule out intracerebral hemorrhage. Demarcated infarcts were found in 
Table II. Distribution of stroke in the different groups.

\begin{tabular}{lcccc}
\hline Infarct & Total, $\mathrm{n}$ & Group A, $\mathrm{n}$ & Group B, $\mathrm{n}$ & Group C, $\mathrm{n}$ \\
\hline Anterior circulation infarct & 36 & 20 & 2 & 14 \\
Posterior circulation infarct & 80 & 46 & 22 & 12 \\
Watershed infarct & 4 & 2 & 2 & - \\
\hline
\end{tabular}

Table III. Time to initiation of treatment with tirofiban (hours).

\begin{tabular}{lcccc}
\hline Statistic & Total population & Group A & Group B & Group C \\
\hline Mean & 9.5 & 11.6 & 9.3 & 3.6 \\
Median & 4.5 & 4.3 & 7.0 & 2.5 \\
\hline
\end{tabular}

Table IV. Frequency of bleeding in the groups.

\begin{tabular}{|c|c|c|c|c|c|c|}
\hline Group & Total bleeding, n (\%) & $\mathrm{HI} 1, \mathrm{n}(\%)$ & $\mathrm{HI} 2, \mathrm{n}(\%)$ & $\mathrm{PH} 1, \mathrm{n}(\%)$ & $\mathrm{PH} 2, \mathrm{n}(\%)$ & $\mathrm{SICH}, \mathrm{n}(\%)$ \\
\hline Group A & - & - & - & - & - & - \\
\hline Group B & $6(23.1)$ & - & - & $6(23.1)$ & - & - \\
\hline Group C & $2(7.7)$ & - & - & $2(7.7)$ & - & - \\
\hline Total & $8(6.7)$ & - & - & $8(6.7)$ & - & - \\
\hline
\end{tabular}

HI, hemorrhagic infarction; $\mathrm{PH}$, parenchymal hematoma; SICH, symptomatic intracerebral hemorrhage.

32 patients in the imaging studies, while another 24 patients had early infarct signs.

A total of $93.3 \%$ of the patients underwent vascular imaging with CTA, MRA or DSA. Transcranial duplex sonography was carried out in two cases. Sixty-eight patients $(56.7 \%$ of the total population) had a complete or nearly complete vessel occlusion.

Clinical outcomes and complications. The application of tirofiban was recommended for a duration of $24 \mathrm{~h}$; however, the infusions were continued until a stable situation had been established in 12 cases. Furthermore, a second dose was administered in cases of fluctuating symptoms, which significantly extended the total application time. The mean treatment duration was $30.5 \mathrm{~h}$ (range, $4-106 \mathrm{~h}$ ). The mean cumulative dose was $15.7 \mathrm{mg}$ tirofiban (range, $2.5-71.9 \mathrm{mg}$ ). The infusion had to be terminated prematurely in 18 patients due to complications. Two patients exhibited a contrast-medium extravasation injury in the basal ganglia by CT imaging following thrombolysis; in these cases, the infusion was terminated due to the potential masking of a bleed by the contrast agent. Four patients developed a large space-occupying infarction and had to undergo a hemicraniectomy. In two cases there were concerns whether the treatment should continue, since the patients were suffering from colon cancer. Thrombocytopenia occurred in two cases. Two patients suffered from hematemesis and four patients had controlled parenchymal bleeding at the end of the CT.

The time from symptom onset to tirofiban administration showed considerable variation and ranged from 45 to $72 \mathrm{~h}$; this was particularly due to the fact the patients in
Table V. Arterial recanalization rate for patients diagnosed with vascular occlusion.

\begin{tabular}{lc} 
Therapy & Recanalization, $\mathrm{n}(\%)$ \\
\hline Tirofiban monotherapy, $\mathrm{n}=8$ & $2(25)$ \\
IV thrombolysis, $\mathrm{n}=8$ & $4(50)$ \\
IA thrombolysis, $\mathrm{n}=16$ & $14(88)$ \\
IV and IA thrombolysis, $\mathrm{n}=2$ & $0(0)$ \\
Embolectomy, $\mathrm{n}=4$ & $2(50)$ \\
Early CEA, $\mathrm{n}=2$ & $2(100)$ \\
Total, $\mathrm{n}=40$ & $24(60)$
\end{tabular}

IV, intravenous; IA, intra-arterial; CEA, carotid endarterectomy.

group A were treated only with tirofiban and the fluctuation in the symptoms was noticeable. In 10 patients, the time of symptom onset could not be determined as the patient woke up with symptoms. The median time represented the time that similar treatments were started in groups $\mathrm{A}$ and $\mathrm{C}$ (Table III).

In the total population, only eight $(6.7 \%)$ out of all the patients suffered hemorrhage. All hemorrhages were classified as parenchymal bleeding without symptomatic bleeding. Table IV shows the frequency of bleeding in each group.

A total of 14 patients $(11.7 \%)$ experienced extracerebral bleeding complications. Three, five and two patients suffered 
A

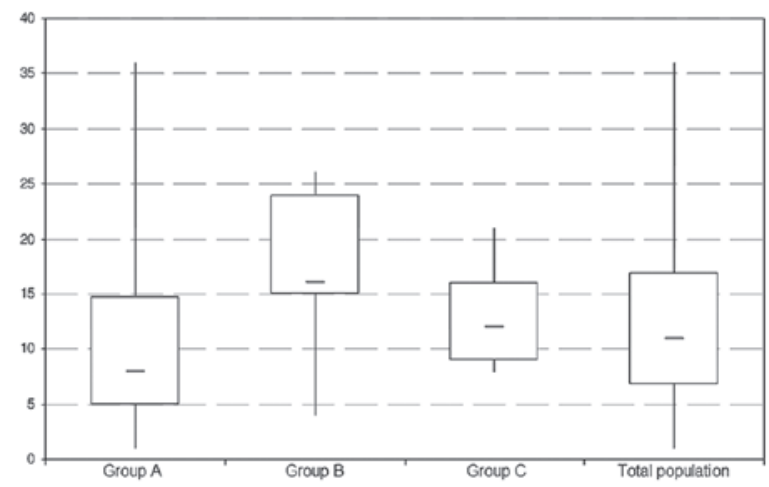

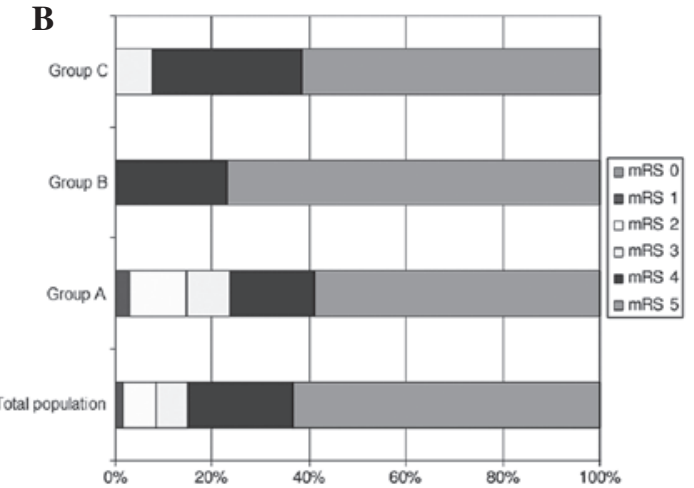

Figure 1. Stroke severity in each group at admission; (A) National Institutes of Health Stroke Scale, (B) modified Rankin Scale (mRS).

A

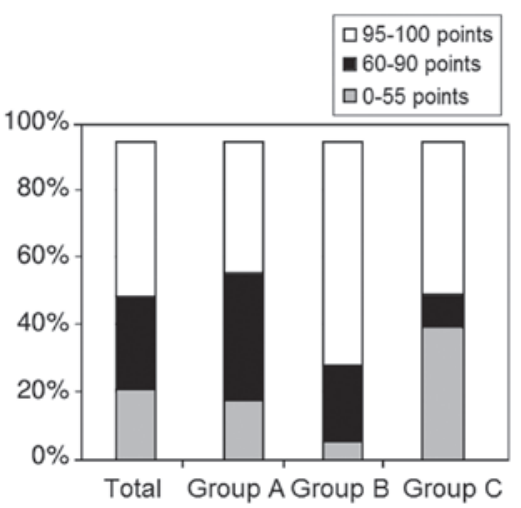

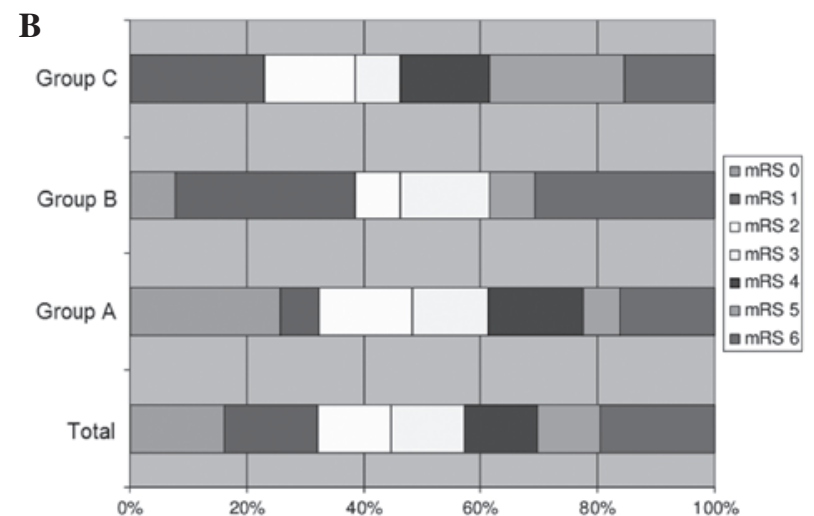

Figure 2. Outcome of patients in each group at least three months after stroke: (A) Barthel index, (B) modified Rankin Scale (mRS).

from gastrointestinal hemorrhage, abdominal wall hematoma and compartment syndrome following hemorrhage in the thigh, respectively. None of the patients required a blood transfusion. Two out of the 14 patients had received tirofiban following systemic lysis therapy with $\mathrm{tPA}$, while the remaining 12 patients were treated in group A. One patient in group A was observed to have clinically insignificant thrombocytopenia.

A complete or nearly complete vessel occlusion was detected in 68 patients, 38 of whom were subjected to transcranial duplex sonography. Twenty-four patients underwent recanalization of the occluded vessel (Table V).

Stoke severity. To assess the severity of the stroke, the patients were assessed by the mRS and NIHSS at admission, as shown in Fig. 1. The median NIHSS was 11 points in the overall patient population, eight points in group A, 16 points in group B and 12 points in group C. Overall, the variation was large, but most cases were serious.

A total of 16 patients (six in group A, eight in group B and two in group C) succumbed during the hospital stay; the mortality rate was $13.3 \%$ (8.8\% for group A, 30.7\% for group B and $7.7 \%$ for group C) in the acute phase. These 16 patients had an mRS of five at admission. Forty-eight of the 120 patients or their relatives were interviewed. The follow-up period was between three and 14 months after the stroke, and the BI and mRS were queried. The results are shown in Fig. 2.

A favorable outcome (mRS, 0-2) during the first three months after the stroke was only observed in $43.3 \%$ of the patients (44.1\% in group A, $46.7 \%$ in group B and $36.4 \%$ in group $\mathrm{C}$ ). The mean BI was 72.3 points in group $\mathrm{A}, 84.4$ points in group $\mathrm{B}, 56.8$ points in group $\mathrm{C}$ and 71.0 points in the total patient population.

\section{Discussion}

In this retrospective study, it was shown that, in certain cases, the gpIIb/IIIa antagonist tirofiban could be used for the treatment of AIS. The present study was retrospective, however, not controlled, and considered various treatment strategies simultaneously; there is therefore an urgent need to confirm the results with larger, prospective, controlled studies.

In the present study, the outcomes of group A and group $\mathrm{C}$ were better than group B. No patients in group A and only two patients in group $\mathrm{C}$ had asymptomatic hemorrhages. A follow-up study comparing rtPA (dosage, $0.6 \mathrm{mg} / \mathrm{kg}$ ) plus eptifibatide with standard lysis is in process. This may then become a phase III efficacy study (13).

Notable results have also been found in experimental stroke studies in rodents. In a study by Kleinschnitz et al (14), a dose-dependent association was found between the risk of intracerebral hemorrhage and the use of anti-mouse gpIIb/IIIa $\mathrm{F}(\mathrm{ab})_{2}$ fragments at doses resulting in a receptor blockade of $>95 \%$, but not at doses resulting in a receptor blockade of $67.8 \%$. Choudhri et al (15) found significant bleeding following the administration of the non-peptide substance SDZ GPI 562 at maximum doses in a mouse model of AIS. Subsequent 
to the administration of lower doses, a significantly smaller infarct volume than expected was observed by staining with triphenyltetrazolium chloride. Other studies in experimental stroke models in guinea pigs and squirrel monkeys with the non-peptide gpIIb/IIIa blocker FK419 revealed no bleeding complications, but showed reduced infarct volume as an indication of their effectiveness $(16,17)$.

The gpIIb/IIIa receptor (integrin aIIbb3) has the same $\beta 3$ subunit as the vitronectin receptor (integrin $\alpha v \beta 3$ ), which is present on resting endothelial cells in small numbers; however, the expression of $\alpha v \beta 3$ is upregulated in response to angiogenic stimuli, such as hypoxia, transforming growth factor- $\beta 3$ and thrombin, as they occur in the context of regional cerebral ischemia. The expression of the vitronectin receptor on endothelial cells is responsible for the adhesion of monocytes to the endothelium, conveys permeability to the blood-brain barrier and, with vascular endothelial growth factor, contributes to the proliferation and migration of inflammatory cells into the perivascular tissue during angiogenesis $(18,19)$. The binding of gpIIb/IIIa receptor blockers to the vitronectin receptor affects the permeability of the blood-brain barrier and thus influences the occurrence of intracerebral hemorrhage. A dose-dependent study of the effects of gpIIb/IIIa blockers on activated endothelial cells may provide further insight.

While the link between fibronectin receptor interference and the occurrence of intracranial bleeding (ICB) is currently more of a theoretical nature, the favorable association between vascular occlusion and reperfusion subsequent to ICB has been previously shown (20). The use of biomarkers in the blood-brain barrier enables the prediction of intracranial hemorrhagic complications following stroke and particularly subsequent to thrombolysis with the administration of an additional therapeutic agent. Specifically, matrix metalloproteinase-9, cellular fibronectin, $\mathrm{S} 100 \beta$ and glial fibrillary acidic protein have been shown to facilitate the prediction of intracranial hemorrhage (21).

Biomarkers could also be used to study the different gpIIb/IIIa antagonists with regard to bleeding complications. Mangiafico et al (22) described 21 patients with AIS who underwent an aggressive treatment regimen consisting of IV tirofiban for 24 to $48 \mathrm{~h}$, IV heparin, local lysis with urokinase and, in the majority of patients, percutaneous transluminal angioplasty. It should be noted, however, that the comparability is limited due to low patient numbers. A previous study (23) investigated the combination of tirofiban with unfractionated IV heparin (UFH) or with IV rtPA in the treatment of acute stroke. Junghans et al (23) prospectively studied 18 patients within $24 \mathrm{~h}$ after the onset of stroke symptoms; the patients were initially treated with UFH, with a target activated partial thromboplastin time of 50-70 sec, and then tirofiban at the dosage recommended in the Platelet Receptor Inhibition in Ischemic Syndrome Management in Patients Limited by Unstable Signs and Symptoms study (24) for $\sim 46 \mathrm{~h}$. Although no major intracerebral hemorrhage was observed in the study, only a low recanalization rate of $25 \%$ was obtained.

Both tirofiban and heparin possess thrombolytic properties. The rational behind the treatment is associated with the time it takes for an endogenous mechanism mediated by the endothelial cells to extend the effects of the thrombolytic therapy to prevent the occurrence of further thrombi and the reocclusion of re-opened vessels. In addition to the narrow time window, sole rtPA administration causes easy vessel re-opening in $\sim 1 / 3$ re-occluded cases (25). In group B of our work, the vessel re-opening still exists despite the major tribes limit the microcirculation.

In the present study, the long-term outcome of the patients in group A was not as promising as that observed for the SaTIS population; the SaTIS trial is the only previous study of tirofiban in a large cohort of stroke patients (5). Furthermore, in the present study the mean $\mathrm{BI} \geq 3$ months after stroke was 71 points (median, 90).

Overall, the present study has demonstrated the safety of tirofiban in monotherapy or in combination with various recanalization methods in the treatment of patients with AIS. Due to the retrospective, non-controlled study design and the relatively low numbers of patients with heterogeneous treatment approaches, these data must be interpreted with caution; however, they give a good insight into the safety of the use of tirofiban in clinical practice.

\section{References}

1. Suwanwela NC, Phanthumchinda K and Likitjaroen Y: Thrombolytic therapy in acute ischemic stroke in Asia: The first prospective evaluation. Clin Neurol Neurosurg 108: $549-552,2006$

2. Nandigam K, Narayan SK, Elangovan S, Dutta TK, Sethuraman KR and Das AK: Feasibility of acute thrombolytic therapy for stroke. Neurol India 51: 470-473, 2003.

3. Matsuo R, Kamouchi M, Fukuda H, et al; FSR Investigators: Intravenous thrombolysis with recombinant tissue plasminogen activator for ischemic stroke patients over 80 years old: The Fukuoka Stroke Registry. PLoS One 9: e110444, 2014.

4. Kumar S, Rajshekher G and Prabhakar S: Platelet glycoprotein IIb/IIIa inhibitors in acute ischemic stroke. Neurol India 56: 399-404, 2008

5. Siebler M, Hennerici MG, Schneider D, et al: Safety of Tirofiban in acute Ischemic Stroke: the SaTIS trial. Stroke 42: 2388-2392, 2011.

6. Ciccone A, Abraha I and Santilli I: Glycoprotein IIb-IIIa inhibitors for acute ischemic stroke. Stroke 38: 1113-1114, 2007.

7. Haerten K, Krabbe C and Raiber M: Efficacy and safety of treatment of acute ischemic stroke with glycoprotein IIb/IIIa receptor blocker in routine clinical practice. Dtsch Med Wochenschr 129: 607-610, 2004 (In German).

8. Bogousslavsky J and Leclerc JR: Platelet glycoprotein IIb/IIIa antagonists for acute ischemic stroke. Neurology 57 (5 Suppl 2): S53-S57, 2001

9. Mazighi M, Meseguer E, Labreuche $\mathrm{J}$ and Amarenco P: Bridging therapy in acute ischemic stroke: A systematic review and meta-analysis. Stroke 43: 1302-1308, 2012.

10. Brilakis ES, Banerjee S and Berger PB: Perioperative management of patients with coronary stents. J Am Coll Cardiol 49: 2145-2150, 2007.

11. Banks JL and Marotta CA: Outcomes validity and reliability of the modified Rankin scale: Implications for stroke clinical trials: A literature review and synthesis. Stroke 38: 1091-1096, 2007.

12. Hacke W, Kaste M, Fieschi C, et al: Randomised double-blind placebo-controlled trial of thrombolytic therapy with intravenous alteplase in acute ischaemic stroke (ECASS II). Second European-Australasian Acute Stroke Study Investigators. Lancet 352: 1245-1251, 1998.

13. Pancioli AM, Broderick T, Brott T, et al; CLEAR Trial Investigators: The combined approach to lysis utilizing eptifibatide and rt-PA in acute ischemic stroke: the CLEAR stroke trial. Stroke 39: 3268-3276, 2008.

14. Kleinschnitz C, Pozgajova M, Pham M, Bendszus M, Nieswandt B and Stoll G: Targeting platelets in acute experimental stroke: Impact of glycoprotein Ib, VI, and IIb/IIIa blockade on infarct size, functional outcome, and intracranial bleeding. Circulation 115: 2323-2330, 2007. 
15. Choudhri TF, Hoh BL, Zerwes HG, et al: Reduced microvascular thrombosis and improved outcome in acute murine stroke by inhibiting GP IIb/IIIa receptor-mediated platelet aggregation. J Clin Invest 102: 1301-1310, 1998.

16. Moriguchi A, Maeda M, Mihara K, Aoki T, Matsuoka N and Mutoh S: FK419, a novel nonpeptide GPIIb/IIIa antagonist, restores microvascular patency and improves outcome in the guinea-pig middle cerebral artery thrombotic occlusion model: comparison with tirofiban. J Cereb Blood Flow Metab 25: 75-86, 2005.

17. Maeda M, Moriguchi A, Mihara K, et al: FK419, a nonpeptide platelet glycoprotein IIb/IIIa antagonist, ameliorates brain infarction associated with thrombotic focal cerebral ischemia in monkeys: comparison with tissue plasminogen activator. J Cereb Blood Flow Metab 25: 108-118, 2005.

18. Coller BS: Binding of abciximab to alpha V beta 3 and activated alpha $M$ beta 2 receptors: with a review of platelet-leukocyte interactions. Thromb Haemost 82: 326-336, 1999.

19. Murphy JF, Bourdet JC, Wyler B, et al: The vitronectin receptor (alpha v beta 3 ) is implicated, in coorperation with P-selectin and platelet-activating factor, in the adhesion of monocytes to activated endothelial cells. Biochem J 304: 537-542, 1994.

20. MolinaCA,Alvarez-Sabín J,Montaner J,etal: Thrombolysis-related hemorrhagic infarction: a marker of early reperfusion, reduced infarct size, and improved outcome in patients with proximal middle cerebral artery occlusion. Stroke 33: 1551-1556, 2002.
21. Montaner J, Molina CA, Monasterio J, et al: Matrix metalloproteinase-9 pretreatment level predicts intracranial hemorrhagic complications after thrombolysis in human stroke. Circulation 107: 598-603, 2003.

22. Mangiafico S, Cellerini M, Nencini P, Gensini G and Inzitari D: Intravenous glycoprotein IIb/IIIa inhibitor (tirofiban) followed by intra-arterial urokinase and mechanical thrombolysis in stroke. AJNR Am J Neuroradiol 26: 2595-2601, 2005.

23. Junghans U, Seitz RJ, Aulich A, Freund HJ and Siebler M: Bleeding risk of tirofiban, a nonpeptide GPIIb/IIIa platelet receptor antagonist in progressive stroke: an open pilot study. Cerebrovasc Dis 12: 308-312, 2001.

24. Platelet Receptor Inhibition in Ischemic Syndrome Management in Patients Limited by Unstable Signs and Symptoms (PRISM-PLUS) Study Investigators: Inhibition of the platelet glycoprotein IIb/IIIa receptor with tirofiban in unstable angina and non-Q-wave myocardial infarction. N Engl J Med 338: 1488-1497, 1998.

25. Alexandrov AV and Grotta JC: Arterial reocclusion in stroke patients treated with intravenous tissue plasminogen activator. Neurology 59: 862-867, 2002. 